\title{
Public Flue Smoke Treatment Unit in Residential Building Based on Internet of Things
}

\author{
Hanlei Du \\ North China Electric Power University, Baoding, 071000, China
}

Keywords: Internet of Things, secondary treatment device,Automatic Alarming Device

\begin{abstract}
Nowadays the air pollution is increasingly serious. PM10, PM2.5 and other major pollution comes from various sources, in which one of the important sources is the emission of cooking smoke. Therefore, the control of sources is quite important to emission reduction. With the development of cities, more and more high-rise residential buildings are built. However, most public flues in such buildings are lack of secondary treatment devices of smoke. This paper designs a concentrated secondary treatment device of public flue in residential building to realize the purpose of energy conservation and emission reduction.
\end{abstract}

\section{Overview}

This device is installed at the exit of flue, which is equivalent to a filter installed at the exit. Since household smoke exhaust has made primary treatment on smoke, this device will do secondary treatment to reduce the pollutant. It adopts direct and low cost physical filter with three layers of filter screen. It adds a funnel cap on the device to rotate by making use of high altitude wind to form lower pressure, stabilize smoke upper emission and reduce flowing backward.

The first layer of filter screen is used for filtering residual oil. It adopts nonwoven. Oil filtering nonwoven has the characteristics of good air permeability, good oil absorbency, low cost and non-hydrophilicity. It will not cake after absorbing oil. After experiment, it has been proved that oil filtering nonwoven has good adsorption to oil drops in smoke.

Smoke will enter the second layer of filter screen after passing through nonwoven. The second layer adopts porous activated carbon. Activated carbon has numerous micropores with huge specific surface area, which can effectively remove chromaticity, foul smell and pollutant particle. The experiment shows that porous activated carbon can filter pm10 particle and reduce the occupation of pm10 particle to the third layer of filter screen in the filter device.

The third layer is mainly used for filtering pm2.5 particle. It adopts a new material - Vasional stone. It is an interior decoration material to purify air bringing negative charge to absorb pm2.5.

Three layers of filter screen of the device adopt drawer type installation. Filter screen can be installed on the fixed groove which can be drawn out horizontally. It can be drawn out together with the fixed groove directly when it shall be replaced or cleaned, so the replacement and cleaning is easy. Its model is shown as follows:

A funnel cap is added on the top of the filter device, which can rotate by making use of high altitude wind to form lower pressure, stabilize smoke upper emission and reduce flowing backward. Besides, it does not consume electric energy, reflecting the good performance of energy conservation and environmental protection of the filter device. 

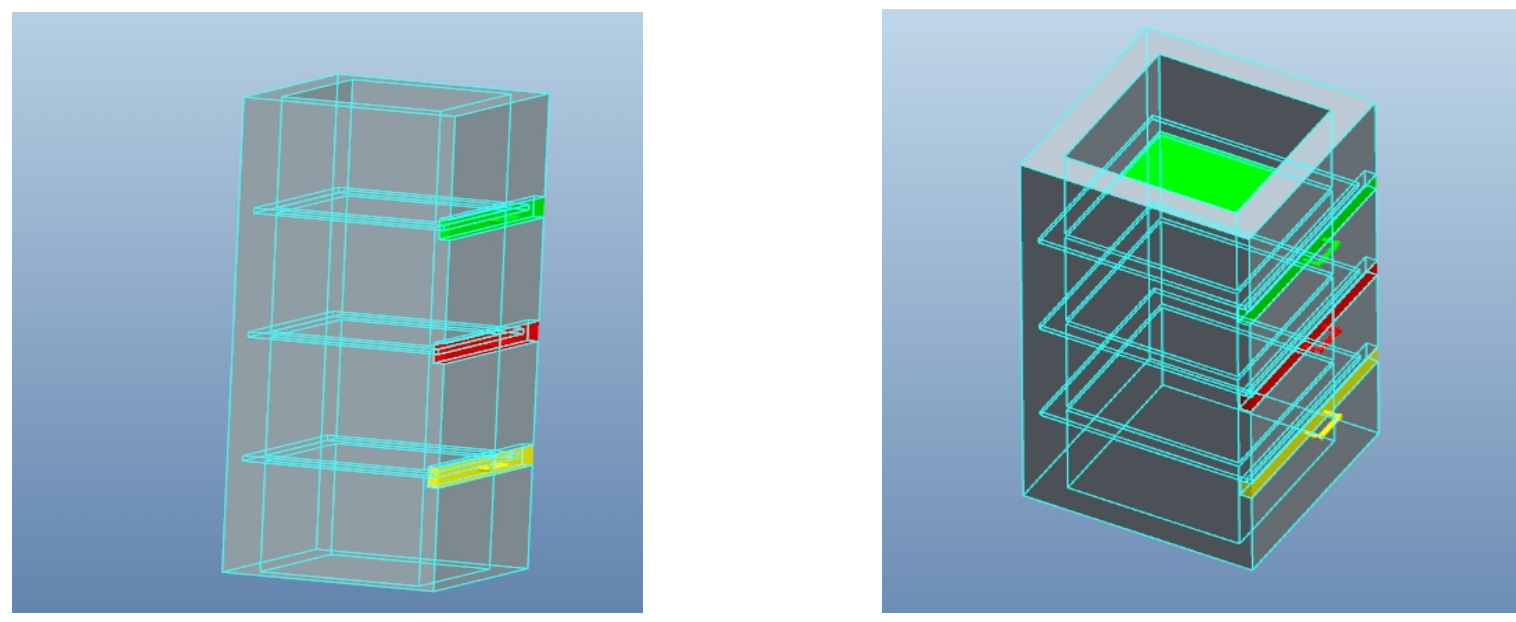

\section{Automatic Alarming Device}

The scientific nature of this device is that it makes use of single chip computer and sensor to do Internet of Things information management in the living quarter and adopts automatic alarming device to inform the property management personnel to replace the filter screen. A pressure sensor is installed at the bottom of the fixed groove of each layer of filter screen. When any layer of filter screen is drawn out to install a new one, the sensor will regard the weight of the new filter screen as the basic value. Since every type of filter screen has its own filter extremity, when the filter capacity is reduced or is impeding the upward emission of smoke, the substance on the filter screen will add certain weight on the filter screen. As long as we have known this added weight through experiment, the sensor will send alarm to the computer of property management through single chip computer to inform of replacement once the added weight reached such value. Three sensors are connected with each other though STC89C51 single chip computer to do common management and signal transmission. It will detect the service life of filter screen automatically and remind the property management personnel of replacement. The device combines with Internet of Things with the characteristic of highly intelligent.

\section{Experimental Analysis}

Many experiments confirm that the device can filter out about seventy percent of PM10 and about fifty-five percent of PM2.5, which means it can achieved a higher level of filtration in a low price.

\section{Economic and Technological Analysis}

Since the device adopts direct physical adsorption and filtering to do secondary treatment, which is practical and feasible. Materials used by it are in low cost except for Vasional stone. If the replacement fee of filter screen is undertaken by property management fee paid by residents, the expense is calculated as follows:

Since nonwoven has low cost and wide scope of application, which is not influenced by the market and supply-demand relationship to a large extent, we hereby assume that nonwoven is $15 \mathrm{Yuan} / \mathrm{m} 2$ which shall be replaced monthly.

We select high quality ultra-strong adsorption activated carbon, which shall be replaced every half a year. The manufacturer estimates that 270 Yuan of product can be used for 5 years.

Vasional stone is a new material with high cost in recent years due to market requirement. However, with the improvement of production technology, the price will reduce to a large extent after several years. It is predicted to be $1,000 \mathrm{Yuan} / \mathrm{m} 2$ three years later. Its thickness can be used to replace for 5 times, which shall be replaced every 2 years.

It is predicted to be used by 40 households in 20 stories of a building.

After careful calculation, if it is put into use, each household shall only pay 10.85 Yuan every year (paid in the property management fee). It has high cost performance with a great help to improve the environment of the living quarter and even the city. 
Therefore, relative to air pollution control, this device is quite feasible and economical in material, construction, installation and replacement.

\section{Summary}

With the urbanization progress, more and more high-rise residential buildings are built. However, one residential building only has one or two public flues. If a public flue smoke treatment device is installed at the exit of each flue, it can control the source of air pollution and purify the air with practical significance of energy conservation and emission reduction. The construction fee will be borne by the property management company and the residents will pay property management fee to undertake the filter screen replacement fee. The expense of each household is very little.It will make a difference in controlling pollution source and making the air clean.If you follow the "checklist" your paper will conform to the requirements of the publisher and facilitate a problem-free publication process.

\section{References}

[1] Vibration and Noise Source Identification of Certain Type of Extractor Hood, Li Jiaqing, Chen Jin, Yang Chao, Xue Weifei, Dong Guangming, National Key Laboratory of Mechanical System and Vibration, Shanghai Jiaotong University, Shanghai, 200240

[2] A Technical Discussion on the Smoke Treatment of Catering Business, Luo Shuren, Foshan, 528000

[3] A Discussion on the Technical Performance of Smoke Purification Device of Catering Business, Jiang Changmin, Shanghai Academy of Environmental Sciences, 200233

[4] Automatic Control Technology, China Electric Power Press, Edition 2

[5] National Catering Business Smoke Emission Standards (Trial) GWPB 5-2000

[6] Noise Limit of Household and Electric Apparatus of Similar Use, issued by General Administration of Quality Supervision and Standardization Administration of the People's Republic of China

[7] Xin-min pan, Wang Yanfang. Microcomputer control technology. People's posts and telecommunications publishing house. 1999

[8] Li Chaoqing. Single chip microcomputer principle and application technology. Nankai university press 